\title{
Análisis de los componentes productivos de la hacienda El Puente
}

\section{Analysis of the productive components of the El Puente farm}

María Fernanda Urbano Piñaloza. ${ }^{1}$, Fabián Augusto Almeida López. ${ }^{2}$, Paula Alexandra Toalombo Vargas. ${ }^{3}$ \& Julio Cesar Benavides Lara. ${ }^{4}$

\begin{abstract}
.
DOI: https://doi.org/10.33262/visionariodigital.v4i3.1397

At Hacienda El Puente, Riobamba canton, Chimborazo province, the economic elements of this estate were determined, with a duration of 60 days. The collected data were subjected to statistical analyzes such as: standard deviation, frequency histograms, mean and the economic study through the benefit / cost indicator. For the economic and technical analysis, production costs were considered, based on fixed costs, variable costs, cost structure and profitability. Regarding the part of production that intervened in 2017 at Hacienda el Puente are the variable costs: sanitary aspect (\$ 5178.81), feeding and supplementation (\$16070.68), reproduction and livestock improvement $(\$ 3,265.45)$, occasional labor $(\$ 1932.00)$, transportation (\$ 3324.70), and fixed costs: administrative cost $(\$ 3293.72)$, permanent labor $(\$ 14,400.00)$, tools (\$ 1,528.23), machinery (\$18,272.00), facilities (\$3808.00), equipment and accessories ( $\$ 4188.95)$. In addition, the income generated by the sale of milk during this year was $\$ 70,978.36$; where, the cost of producing a liter of milk was $\$ 0.49$; its sale is $\$ 0.55$; achieving a net profit of $\$ 0.06$ for each liter of milk sold and a cost benefit of $\$ 1.13$; which shows that for every dollar invested there is a profit of $\$ 0.13$ ctvs. It is recommended to make a better daily record of the expenses that are made in
\end{abstract}

\footnotetext{
${ }^{1}$ Investigador principal.

${ }^{2}$ Magister en Reproducción Animal mención Bovinos de Leche, Ingeniero Zootecnista, Docente de la Escuela Superior Politécnica de Chimborazo, fabian.almeida@espoch.edu.ec

${ }^{3}$ Máster Medicina, Sanidad y Mejora Animal, Ingeniera Zootecnista, Docente de la Escuela Superior Politécnica de Chimborazo, ptoalombo@espoch.edu.ec

${ }^{4}$ Magister en Cadenas Productivas Agroindustriales, Ingeniero Zootecnista, Docente de la Escuela Superior Politécnica de Chimborazo,jbenavides@espoch.edu.ec
} 
this farm, avoiding overlooking minor expenses that affect the correct economic analysis of this Hacienda.

Keywords: Milk production, Economic Evaluation, Dairy cattle, Development, Social economy.

\section{Resumen.}

En la Hacienda El Puente, cantón Riobamba, provincia de Chimborazo, se determinó los elementos económicos esta hacienda, con una duración de 60 días. Los datos recogidos fueron sometidos a los análisis estadísticos como: desviación estándar, histogramas de frecuencia, media y el estudio económico a través del indicador beneficio / costo. Para el análisis económico y técnico se tomó en cuenta los costos productivos, en base a los costos fijos, costos variables, estructura de costos y la rentabilidad. En lo que respecta a la parte de producción que intervinieron en el año 2017 en la Hacienda el Puente son los costos variables: aspecto sanitario (\$ 5178,81), alimentación y suplementación (\$16070,68), reproducción y mejoramiento ganadero (\$ 3265,45), mano de obra ocasional $(\$ 1932,00)$, transporte $(\$ 3324,70)$, y los costos fijos: costo administrativo (\$ 3293,72), mano de obra permanente $(\$ 14400,00)$, herramientas $(\$ 1528,23)$, maquinaria $(\$ 18272,00)$, instalaciones $(\$ 3808,00)$, equipos y accesorios $(\$ 4188,95)$. Además, el ingreso generado por la venta de leche durante este año fue de $\$ 70978,36$; donde, el costo de producir un litro de leche fue de $\$ 0,49$; la venta de este es de $\$ 0,55$; logrando una utilidad neta de $\$ 0,06$ por cada litro de leche vendido y un beneficio costo de $\$ 1,13$; que muestra que por cada dólar invertido se obtiene una ganancia de $\$ 0,13$ ctvs. Se recomienda hacer un mejor registro diario de los gastos que se realizan en esta ganadería, evitando pasar por alto gastos menores que repercuten en el correcto análisis económico de esta Hacienda.

Palabras claves: Producción de leche, Evaluación Económica, Ganado lechero, Desarrollo, Economía social.

\section{Introducción.}

La producción agropecuaria en el país es una fuente de ingresos para el sector primario productivo, esto contribuye al desarrollo de la producción de nuestro país; la producción de leche y sus derivados son productos de gran demanda a nivel mundial, nacional y regional; pero a la vez es deficiente en calidad, debido al bajo nivel de tecnificación de las fincas y al precio de venta de la leche por ello desmotivan al productor.

Aunque no todo son buenas noticias para el productor ya que uno de las amenazas al momento de producir leche es la alta variabilidad del precio de la venta de leche que desmotivan al productor a seguir con esta actividad ya que no existe un valor definido para su venta, el litro de leche está entre 0,30 y 0,48 centavos, es demasiado fluctuante y varía 
según la ganadería, del sector, de su accesibilidad, del mercado internacional, entre muchos otros factores que hacen que los productores no se sientan seguros del retorno de su inversión.

La ganadería hoy en día tiene como propósito lograr que los productores aprovechen el progreso tecnológico; utilizando técnicas adecuadas, dependiendo de las características que presente su ganadería y del sistema de explotación empleado, por ello la elección de la raza bovina es el elemento primordial de un ganadero, seguido de un sistema de manejo adecuado, sistema de alimentación, programas de reproducción y mejora genética y la implementación de un calendario sanitario.

Por otra parte, para aumentar la producción láctea en el ecuador y a su vez promover el consumo de leche por parte de la ciudadanía seria implementando alternativas de publicidad, con políticas y leyes que garanticen el bienestar económico para el pequeño, mediano y grande ganadero; con una estructura fija de los costos de producción. La venta de leche se lo realiza a diario, generando ingresos diarios, el consumo está bien acentuado por los hogares ya que contribuye con la seguridad alimentaria y la nutrición de las personas.

\section{Metodologia.}

Para el desarrollo de la investigación se trabajó bajo los parámetros de una investigación explicativa pues estuvo dirigida a encontrar las causas de los eventos, sucesos o fenómenos sociales, intentando establecer así la relación causa/efecto.

En la fase del planteamiento del problema se empleó la investigación de carácter hipotéticodeductivo, logrando así la formulación de una idea a defender inicial que sirvió de guía en el desarrollo del trabajo. Al hablar de crecimiento económico también lo hacemos de realidad y por ello para su comprensión se utilizó el análisis y la deducción de situaciones y conclusiones, así como de la historia y la lógica.

\section{Localización y duración del experimento.}

La presente investigación se realizó en la Hacienda El Puente, ubicada en el Km 5 de la vía Chambo, cantón Riobamba, provincia de Chimborazo, con una duración de 60 días. La hacienda se encuentra a una altura de $2600 \mathrm{msnm}$.

Tabla 1. Condiciones meteorológicas de la zona.

\begin{tabular}{lc}
\hline Parámetros & Valores promedio \\
\hline Temperatura, ${ }^{\circ} \mathrm{C}$ & 17,30 \\
Precipitación, mm/año & 716,60 \\
Horas luz, año & 165,20 \\
\hline
\end{tabular}


Humedad relativa, $\% \quad 79,50$

Fuente: Elaboración propia.

\section{Unidades experimentales.}

Por tratarse de una investigación de diagnóstico y evaluación, se tomaron en cuenta los factores que intervienen en el proceso de la evaluación económica de la hacienda el Puente, se determinó los costos que interviene en la producción de un litro de leche de acuerdo con la información recolectada en la hacienda por medio de las encuestas y entrevista realizada al propietario de la Hacienda el Puente.

\section{Tratamientos y diseño experimental.}

En la presente investigación no se manejaron tratamientos debido a que es una investigación de diagnóstico, por lo que, no aplica diseño experimental cualquiera.

\section{Medidas experimentales.}

Las variables por estudiar se detallan a continuación:

- Costos variables: Mano de obra ocasional, alimentación, sanidad, producción de leche día, reproducción.

- Costos fijos: Equipos, maquinarias y herramientas, mano de obra permanente, gastos administrativos.

- Organización de costos: Depreciaciones, valoraciones de bienes.

- Rentabilidad: Costo total, unitario, utilidad, beneficio / costo.

\section{Análisis de datos y pruebas de significancia.}

Los resultados experimentales que se analizaron fueron sometidos a los análisis estadísticos: media (x), deviación estándar, histogramas de frecuencia y estudio económico a través del indicador beneficio/costo.

\section{Procedimiento experimental.}

- Para obtener los datos de la investigación se efectuaron diversas visitas a la Hacienda el Puente, a través del análisis descriptivo, en donde la población de estudio está conformada por bovinos productores de leche (raza Jersey); siendo este el universo total y la infraestructura de la hacienda en sí. 
- La encuesta fue elaborada de acuerdo con los parámetros para determinar los costos y evaluar los parámetros productivos de la hacienda el Puente, se empleó la encuesta, y la misma se utilizó y se corrigieron los errores de forma de la misma; además se evaluaron los registros y facturas de compra y venta del periodo de producción 2017 de la hacienda.

\section{Resultados.}

\section{Evaluación de los costos variables en la producción de ganado lechero en la hacienda el puente.}

\section{Sanidad.}

La sanidad en el hato es determinante para obtener una leche de buenas características higiénicas. Esta debe ser más preventiva que curativa. Para lo cual es necesario tener en cuenta lo siguiente: se debe comenzar por tener una vaca saludable y bien nutrida, a la que se le haya aplicado todo el plan de vacunación y vermifugación propio de la zona.

Para evitar la presencia de parásitos externos como moscas y garrapatas, se puede recurrir a productos naturales o biológicos, con el fin de no contaminar la leche con residuos de agroquímicos o utilizar productos que no dejen residuos en la leche.

Las enfermedades que más afectan la calidad de la leche son la mastitis, las fiebres de varios orígenes, la brucelosis, las inflamaciones, abscesos y heridas de los pezones por lo tanto hay que prevenir para evitar encontrarse con estos problemas. En caso de que se presenten enfermedades, se deben aplicar rápidamente los tratamientos indicados, preferiblemente por un Médico Veterinario. Citado en Cabrera, (2004)

El coste del rubro sanidad en la hacienda El Puente, muestra un mayor costo en la compra de antibióticos (\$1716,44); continuado del coste en productos de limpieza $(\$ 667,27)$; en varios (\$ 644,91); en vitaminas (\$563,46); en desinfectantes (\$535,95); en biológicos (vacunas) (\$ $503,76)$; en vermífugos (desparasitantes) $(\$ 143,20)$ y por último el control de calidad de leche $(\$ 60,62)$.

\section{Alimentación.}

La alimentación tiene su influencia sobre la cantidad de leche producida y el contenido de grasa, lactosa, proteína y minerales de la leche. Por regla general cualquier ración que incremente la producción de leche reducirá el porcentaje de grasa.

Una buena alimentación consiste en una ración que llene los requerimientos de crecimiento, producción y reproducción del animal, es decir, que sea adecuada en cantidad y calidad. El 
ganado bovino es un rumiante; por lo tanto, su sistema digestivo está hecho para digerir alimentos como los pastos y forrajes. Citado en Torres, (2009).

Uno de los mayores costos que se determinó en la hacienda dentro del rubro alimentación es en la compra de fertilizantes $\$ 12351,12$; seguido del costo de alimentos balanceados $\$$ 1846,30 ; en herbicidas $\$ 1000,56$; en semillas de pastos $\$ 732,70$ y en suplementos minerales $\$ 140,00$.

\section{Reproducción.}

El costo del rubro reproducción y mejoramiento ganadero en la hacienda El Puente, refleja un alto gasto en la compra de pajillas, siendo el rubro más alto de la hacienda ganadera (\$ $2601,70)$; de igual manera el costo en hormonas $(\$ 465,63)$ y por último en varios rubros $(\$$ 198,12).

Este rubro fue mayor al evaluar los costos de reproducción de animales de la hacienda Monte Carmelo ( $\$ 2849,00)$, esto se puede deber a la capacidad que tienen las vacas de preñarse y no repetir celos reduciendo el número de días abiertos. Citado en Freire (2016)

\section{Mano de obra ocasional.}

El coste del rubro mano de obra ocasional en la hacienda El Puente, se determinó un costo en la contratación de 56 horas a los jornaleros \$ 672,00; de igual manera el costo del establecimiento del programa de manejo de ganaderías \$480,00 al año; este costo debido al servicio de mantenimiento $\$ 480,00$ al año y del costo de transporte del personal $\$ 300,00$.

\section{Evaluación de los costos fijos en la producción de ganado lechero en la hacienda el puente.}

Los costos fijos no varían en relación con el volumen de producción del ganado, y se definirán como costos fijos a la ración de mantenimiento, mano de obra para los animales, mantenimiento de mejoras y otros gastos. En ciertos casos la sanidad y la reposición son gastos variables. Citado en Constanza (2016).

Estos costos se denominan fijos, debido a que permanecen invariables a pesar de los cambios en el nivel de producción durante el año, o sea que se mantienen constantes así la producción disminuya o aumente. Citado en Mendoza (2010).

En el cuadro 2 se muestran los costos fijos de la hacienda. 
Tabla 2. Costos fijos de la hacienda el puente

\begin{tabular}{lr}
\hline Rubro & \multicolumn{1}{c}{ Costo, $\$$} \\
\hline Gastos administrativos & 3293,72 \\
Mano de obra permanente & 14400,00 \\
Herramientas & 1528,23 \\
Maquinaria & 6029,1 \\
Valoración y depreciación & 3808 \\
Equipos & 4188,95 \\
\hline Total \$ & 33248,00 \\
\hline
\end{tabular}

Fuente: Registros de la hacienda el Puente, (2018).

Varios factores están incluidos dentro de estos costos como las edificaciones e instalaciones, el personal administrativo, la maquinaria, entre otros, también se conoce como "capacidad instalada".

Las infraestructuras de una explotación ganadera tienen una vida promedio de 10 años, siempre y cuando se realice el mantenimiento de las instalaciones, además este rubro por lo general los productores no lo consideran porque se los construye con materiales propios de la zona y se abaratan costos.

\section{Inventario de animales}

En la Hacienda el Puente se pudo contabilizar un total de 50 animales (cuadro 3), 23 vacas en producción, 9 vacas secas, 17 terneras y 1 toro.

Tabla 3. Inventario de animales de la hacienda el puente

\begin{tabular}{lcc}
\hline \multicolumn{1}{c}{ Categoría } & Número & Porcentaje \% \\
\hline Vacas en producción & 23,00 & 46,00 \\
Vacas secas & 9,00 & 18,00 \\
Terneras & 17,00 & 34,00 \\
Toros & 1,00 & 2,00 \\
\hline Total & 50,00 & \\
\hline
\end{tabular}

Fuente: Registros de la hacienda el Puente, (2018).

Si se porcentualiza el hato ganadero de la Hacienda el Puente, la mayoría del hato está constituido con el $46 \%$ lo constituyen las vacas en producción, seguidos de un $34 \%$ de las terneras, $18 \%$ vacas secas y un $2 \%$ de toros. Dichos porcentajes son aceptables, debido a 
que el principal ingreso de una producción lechera se basa precisamente en los litros de leche que producen diariamente las vacas en producción.

\section{Indicador beneficio costo.}

Para interpretar los resultados del beneficio/costo, es cuando los resultados son mayores que 1 , significa que los ingresos netos son superiores a los egresos netos. En otras palabras, los beneficios (ingresos) son mayores a los sacrificios (egresos) y, en consecuencia, el proyecto generará ganancia a una comunidad y traerá consigo un beneficio social. Citado en Encalada, (2015).

En lo que respecta al indicador beneficio costo de la hacienda El Puente, se pudo determinar un valor de 1,13 (cuadro 4); lo que quiere decir que por cada dólar invertido se obtiene una ganancia de $\$ 0,13$.

\section{Punto de equilibrio.}

El valor de punto de equilibrio de la hacienda ganadera El Puente es de 50710,24; en litros de leche producidos es de 101420,48; y en toneladas de leche es de 104,67 determinándose un valor de 132,06 ton de leche al año producida; es decir la hacienda tiene una ganancia de 27,39 ton de leche al año. Lo que hace referencia a la situación de ventas, donde los costos fijos y los costos variables se encuentran cubiertos.

Con lo determinado anteriormente, podemos mencionar que una empresa logra cubrir sus costos al incrementar sus ventas, la cual logrará ubicarse por encima del punto de equilibrio obteniendo beneficio efectivo para la empresa.

\section{Cuál es el costo para producir 1 litro de leche.}

Una vez determinado los costos fijos y los costos variables, se pudo determinar el costo de producir un litro de leche en la Hacienda El Puente, estableciendo el valor de \$ 0,49 (cuadro 4), este rubro se lo obtuvo dividiendo los egresos totales de la finca para la producción total de leche producida durante el año; el costo de venta del litro de leche producido en la hacienda, es de $\$ 0,55$; con lo que se obtuvo una utilidad neta de $\$ 0,06$ por cada litro de leche entregada.

Tabla 4. Evaluación económica de la hacienda el Puente.

\begin{tabular}{cc}
\hline Rubro & Valor total \\
\hline Ingresos, \$ & 70978,36
\end{tabular}




$\begin{array}{lc}\text { Egresos, } \$ & 63019,64 \\ \text { Producción, leche/año } & 127596,92 \\ \text { Costo, leche/litro \$ } & 0,49 \\ \text { Utilidad, leche/litro } & 0,06 \\ \text { Beneficio costo } & 1,13 \\ \text { Punto de equilibrio } & 50710,24 \\ \text { Punto de equilibrio, litros } & 101420,48\end{array}$

Fuente: Registros de la hacienda el Puente, (2018).

\section{Producción de leche.}

En la provincia de Chimborazo alrededor de 56.659 cabezas de ganado son las que se ordeñan; ya sea de forma manual o mecánica; recalcando que, según la información obtenida, la mayoría de los ganaderos ordeñan a sus vacas de manera manual. Citado en Guamán, (2016).

En cuanto a la producción de leche de la hacienda el Puente, durante el año se produjeron 127596,92 litros, durante el transcurso del año no hubo una producción homogénea, esto debido a que no todas las vacas se encuentran en el mismo estado fisiológico (lactación), pero en los meses de septiembre 542,70 1, noviembre 509,49 1 y agosto 501,63 1 se lograron los valores más altos en producción láctea.

Para los meses de abril 320,06 litros y mayo 378,12 litros se pudo evidenciar una baja en la producción de leche al analizar los registros productivos.

La venta total de la producción de leche se la comercializa directamente a una empresa privada, la cual paga el litro de leche de acuerdo con varios parámetros de calidad, por lo que este valor varía considerablemente en cada entrega, pero de manera general el litro de leche se encuentra entre $\$ 0,50$ y 0,55. El volumen de producción de leche mensual se describe en el cuadro 5.

Tabla 5. Volumen de producción de leche mensual de la hacienda el puente.

\begin{tabular}{lcc}
\hline \multicolumn{1}{c}{ Mes } & Media & Desviación típica \\
\hline Enero & 443,79 & 182,29 \\
Febrero & 494,56 & 242,39 \\
Marzo & 453,78 & 263,51 \\
Abril & 320,06 & 77,03 \\
Mayo & 378,12 & 208,95 \\
Junio & 462,08 & 239,09 \\
Julio & 383,49 & 194,75
\end{tabular}




\begin{tabular}{lll} 
Agosto & 501,63 & 261,60 \\
Septiembre & 542,70 & 392,70 \\
Octubre & 451,26 & 342,23 \\
Noviembre & 509,49 & 360,22 \\
Diciembre & 490,72 & 206,53 \\
\hline Total & 447,71 & 254,03 \\
\hline
\end{tabular}

Fuente: Registros de la hacienda el Puente, (2018).

\section{Discusión:}

Analizados los resultados obtenidos podemos comprobar que uno de los mayores inconvenientes que presentan los bovinos productores de leche, está dado por las diferentes enfermedades reproductivas que sobre todo afectan a los parámetros productivos; que influyen en los gastos de producción, y por ende estos aumenten, lo que a la final se traduce en menos réditos económicos para el productor ya que los ganaderos vecinos van a producir más a menor costo. Citado en Fernández (2017).

El costo total en la contratación de mano de obra ocasional fue de \$1932,00; comparados con los costos productivos de la hacienda Monte Carmelo (\$15600,00), esto debido a la permanencia constante del propietario dentro de la hacienda lo que reduce el gasto de contratar gente externa. Citado en Freire (2016).

Los costos en reproducción, constituye conceptualmente como el costo de preñar una vaca por la técnica de inseminación artificial, los costos incluyen la compra de todos los materiales necesarios como las pajuelas, nitrógeno de conservación, guantes de manejo, guantes de látex, entre otros. Citado en Alonso (2016).

El costo total en alimentación fue de \$16070,68; que al comparar con los costos en alimentación de la hacienda Monte Carmelo $(\$ 61643,00)$, se aprecia que esta variación se debe al sistema de manejo de alimentación que se lleva y la capacidad de conversión de los alimentos suministrados de cada uno de los animales. Citado en Freire (2016).

Los costos, cuando las pasturas son la fuente primordial de alimentación, pueden personificar del $42 \%$ al $60 \%$ de los ingresos brutos. Un forraje que cuente con un valor nutricional alto en energía, proteína y minerales, ayuda a que el costo de suplementación se disminuya, siendo esta una relación inversamente proporcional. Citado en Matamoros (2016).

Plan de mejoras recomendado para la hacienda el puente.

La provincia de Chimborazo se caracteriza por ser un potencial en la producción de leche, además de considerar una diversidad en cuanto a volumen de leche producida, que está 
relacionado con el precio de esta, el cual es fluctuante, además este depende en gran parte de la calidad más no del volumen.

La Hacienda EL Puente perteneciente al Ing. Edwin Alzamora, que está conformada por una extensión de 5,5 hectáreas, bajo la administración de sus propietarios, la explotación esta manejado de una manera semi intensiva y por ende el mejoramiento de sus animales es uno de los principales ejes en su manejo productivo; lo que a predispuesto a desarrollar problemas a causa del mismo mejoramiento como: la presencia de afecciones pódales, problemas de mastitis, mayores gastos en sanidad, número mayor de servicios por concepción, ocasionando el descarte de los animales a temprana edad y que los costos productivos aumenten.

Para bajar los gastos y mejorar la producción de la hacienda el Puente, se plantea la ejecución de un plan mejoras orientado no solo a los cambios a realizarse si no a las actividades que conlleva el incremento y mejoramiento de los parámetros productivos; lo que se justifica que al ser una explotación dedicada a la producción de leche se ha dado una mayor importancia para el desarrollo económico y social, el que está encaminado en la contribución de la seguridad alimentaria.

Se proyecta mejorar el sistema productivo en la hacienda el Puente, proponiendo la aplicación de técnicas que estén de acorde a la realidad productiva, seguido de capacitaciones periódicas a los trabajadores y con la participación del propietario acerca de las tecnologías de producción

\section{Acciones por ejecutarse.}

Una vez realizada la evaluación del estado de la Hacienda el Puente, se consideró diversos parámetros para poner en práctica un plan productivo y reproductivo, entre los cuales podemos considerar los siguientes:

\section{Diagnóstico reproductivo de los animales.}

En el cuadro 6 se detalla el diagnostico actual reproductivo del hato ganadero de la hacienda El Puente.

Tabla 6. Diagnóstico reproductivo de los animales

\section{Diagnóstico reproductivo}

Objetivo

Efectuar prácticas para que realizar el diagnóstico reproductivo de los animales de la hacienda el Puente. 
Con los datos conseguidos del aspecto reproductivo del hato

Entorno actual

Acciones para ejecutar

Período de ejecución

Periodicidad

Responsable

Seguimiento / Producto lechero de la hacienda, son bajos por la presencia de muchos días abiertos. Por la presencia de anestro y celos silenciosos en los animales.

Aplicar técnicas adecuadas para la observación de celos en los animales.

Realizar capacitaciones al personal encargado de las vacas para que cumplan esta tarea correctamente.

Realizar chequeos ginecológicos preventivos.

Estas medidas deben empezar a aplicarse inmediatamente después de su aprobación.

Realizar un adecuado manejo de los animales

Diario

Chequeos ginecológicos Trimestral

Contar un macho recelador Diario

Valoración de plan de Trimestral mejoras

El propietario/administrador de la hacienda se encargará de constatar que se dé cumplimiento las acciones planteadas a ejecutarse.

Realizar un informe de las actividades cumplidas y los resultados obtenidos.

Fuente: Registros de la hacienda el Puente, (2018).

\section{Manejo de Instalaciones.}

En el cuadro 7 se especifica el manejo de las instalaciones.

Tabla 7. Manejo de instalaciones

\begin{tabular}{cl}
\hline & Manejo de instalaciones \\
\hline Objetivo & Proporcionar un manejo y mantenimiento oportu \\
& no a las infraestructuras presentes en la Hacienda. \\
& El método de manejo de \\
& explotaciones, así como de la situación de \\
& la producción y alimentación, son causas para \\
& el desarrollo de afecciones pódales repercutiendo \\
& en un egreso financiero para los productores y en \\
& la hacienda El Puente no es la excepción. \\
\hline
\end{tabular}


Se debe utilizar una nutrición eficiente.

Limpieza de patas y pezuñas.

Implementar cronograma de mantenimiento y

Acciones por efectuar un correcto empleo de las instalaciones Aplicar sulfato de cobre en el ingreso del establo para minimizar las afecciones podales que repercuten el estatus productivo de los animales.

Estas medidas deben comenzar a

Período de ejecución aplicarse seguidamente después de su autorización.

Aplicación de sulfato cobre a la entrada del corral

Cada 2 días

Lavado de patas y pezuñas Mensual

Periodicidad

Cronograma de mantenimiento de las instalaciones

Control del sistema de alimentación

Diario

El propietario de la hacienda deberá encargarse

Responsable de examinar que se dé acatamiento de las acciones planteadas a realizarse.

Se debe proceder un informe de las actividades cumplidas y tomar en

Seguimiento / Producto consideración la ocurrencia de las afecciones pódales.

Fuente: Registros de la hacienda el Puente, (2018).

\section{Desarrollo del plan sanitario en los animales.}

En el cuadro 8 se describe el plan sanitario de los animales.

Tabla 8. Desarrollo del plan sanitario en los animales

\begin{tabular}{cl}
\hline \multicolumn{3}{c}{ Plan sanitario en los animales } \\
\hline \multirow{2}{*}{ Objetivo } & Aminorar la aparición de mastitis en los animales, \\
& para aumentar la producción de leche. \\
& Se evidencia un alto costo en el rubro de sanidad, por ello \\
Entorno actual & el elegir toros para inseminación artificial con altos puntajes \\
& en patas y pezuñas, así como en JUI (índice de ubre de \\
\hline
\end{tabular}


la Jersey) y SCS (contenido de células somáticas) de este modo se podrá minimizar los trastornos de enfermedades pódales y mastitis que influyen en la producción y la permanencia de las vacas en el hato bajando el coste en fármacos evitando la eliminación no voluntaria de los animales.

Acciones por efectuar

Período de ejecución

Periodicidad

Responsable

Seguimiento / Producto
Protocolos de higiene de la hacienda establecidos, asegurando mermar la ocurrencia de mastitis en el hato.

Estas medidas deben empezar a aplicarse de inmediato.

Protocolos de higiene

Diario

El propietario de la hacienda deberá inspeccionar que se cumpla.

Mediante un informe de las actividades cumplidas y estar pendiente de la presencia de mastitis, y si se ha reducido la incidencia en el número de animales

Fuente: Registros de la hacienda el Puente, (2018).

\section{Mejoramiento genético.}

A continuación (cuadro 9), se describe el plan de mejoramiento genético.

Tabla 9. Plan de mejoramiento genético.

\begin{tabular}{cl}
\hline \multicolumn{3}{c}{ Plan de mejoramiento genético } \\
\hline Objetivo & Reducir la incidencia de afecciones pódales y \\
& problemas de mastitis \\
& La hacienda presenta problemas de afecciones en las \\
& pezuñas de los animales y la presencia de mastitis en \\
Entorno actual & vacas en producción, lo cual tiende a una baja en la \\
& producción de leche. \\
& Seleccionar toros para inseminación artificial con altos \\
& puntajes en patas y pezuñas, así como en JUI (índice \\
& de ubre de la Jersey) y SCS (contenido de células \\
Acciones por efectuar & somáticas) de esta manera se podría minimizar los \\
& trastornos de enfermedades pódales y mastitis que \\
& influyen en la producción y la permanencia de las \\
\hline
\end{tabular}


vacas en el hato bajando el costo en fármacos evitando el descarte no voluntario de los animales.

Período de ejecución

Responsable

Seguimiento / Producto

Objetivo
Estas medidas se deben cumplir conforme vayan entrando los animales a la reproducción.

El propietario de la hacienda debe considerar lo que desea mejorar.

Propiedad con buena genética con buena producción en calidad y cantidad.

Programa de ganaderías DAIRYLIVE5

Fuente: Elaboración propia.

\section{Conclusiones.}

- En lo que respecta a los componentes que intervienen en los costes de producción durante el año 2017 en la Hacienda El Puente, fueron los costos variables: Alimentación \$16070,68; sanidad \$ 5178,81; transporte \$ 332469; reproducción $\$ 3265,45$; mano de obra ocasional $\$ 1932,00$ y los costos fijos con un total de $\$$ 33248,00 .

- En la hacienda el Puente al realizar el análisis del beneficio costo, este refleja un valor de \$ 1,13 que indica que por cada dólar invertido se obtiene una utilidad de $\$ 0,13$ centavos de dólar, lo que se podría traducir asimismo en una rentabilidad del $13 \%$, esta rentabilidad es buena en particular considerando los problemas por los que atraviesa el sector lácteo y la economía del país.

- Al analizar los distintos componentes se puedo determinar el costo de producir un litro de leche, dándonos un valor estimado de \$ 0,49 centavos; la obtención y venta de leche en la hacienda es de \$ 0,55 centavos por litro, obteniéndose una utilidad neta de \$ 0,06 centavos por cada litro de leche producido y vendido.

\section{Referencias bibliográficas.}

Alonso, V. (2016). Viabilidad técnica y financiera del cambio del sistema convencional al pastoreo rotacional intensivo del hato lechero de la hacienda Santa Elisa, Danlí. p. 8.

Cabrera, M. (2004). Como obtener leche de buena calidad. Recuperado el 12 de Agosto de 2017, de http://www.agronet.gov.co/www/docs_agronet/2005113012633_c\%

Constanza, P. (2016). Impacto económico del manejo de crianza de vaquillas de remplazo de lecheria. Recuperado el 08 de Agosto de 2017, de http://www.engormix.com 
Encalada, I. (2015). Análisis de la rentabilidad de la ganadería lechera del cantón bucay, provinica de guayas. (Tesis de grado. Ingeniero Zootecnista). Universidad de Guayaquil. Facultad de Ciencias Económicas. Guayaquil. pp. 69-76

Fernández, A. (2017). Productividad y rentabilidad de las fincas lecheras del Municipio Rosario, Distrito Perijá, Estado Zulia. Unidad Coordinadora de Proyectos Conjuntos. Facultad de Ciencias Económicas y Sociales, de Agronomía y de Ciencias Veterinarias. La Universidad del Zulia (Mimeo). pp. 132.

Freire, K. (2016). Caracterización del sistema de producción de ganado lechero en la Hacienda Monte Carmelo (Bachelor's thesis, Escuela Superior Politécnica de Chimborazo). pp. $14-77$.

Guamán, S. (2016). El sector agrícola-ganadero y su aporte al desarrollo local de la parroquia quimiag, provincia de chimborazo, durante el período 2014-2015. (Tesis de Grado. Ingeniero Comercial). Universidad Nacional de Chimborazo

Matamoros, D. (2016). Producción de leche bajo sistemas pastoriles. Disponible en línea en http://www.actualidadagropecuaria.com/actagroweb/publicaciones/agosto.2016/HT ML/files/mobile/index.html\#20, Última comprobación el 17/08/2016.

Mendoza, A. (2010). Estructura de costos y estructura del hato. Disponible en:http://www.zoetecnocampo.com/Documentos/costos_hato.htm,documentos, 10 de Junio de 2011. pp. 33 - 37.

Torres, L. (2009). Estudio de prefactibilidad para la implementación de la producción y comercialización de leche cruda en la finca "la floresta". Escuela Politécnica Nacional del Ecuador.

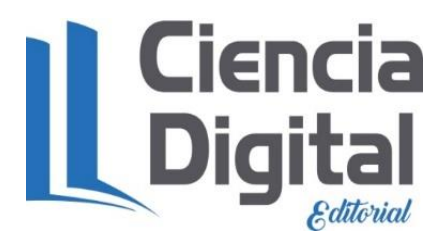


PARA CITAR EL ARTÍCULO INDEXADO.

Urbano Piñaloza, M. F., Almeida López, F. A., Toalombo Vargas, P. A., \& Benavides Lara, J. C. (2020). Análisis de los componentes productivos de la hacienda El Puente. Visionario Digital, 4(3), 247-263. https://doi.org/10.33262/visionariodigital.v4i3.1397

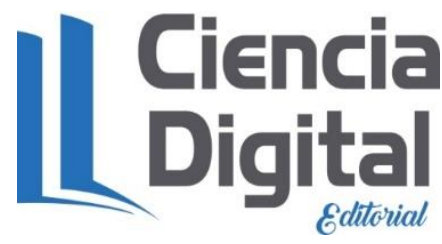

El artículo que se publica es de exclusiva responsabilidad de los autores y no necesariamente reflejan el pensamiento de la Revista Visionario Digital.

El artículo queda en propiedad de la revista y, por tanto, su publicación parcial y/o total en otro medio tiene que ser autorizado por el director de la Revista Visionario Digital.
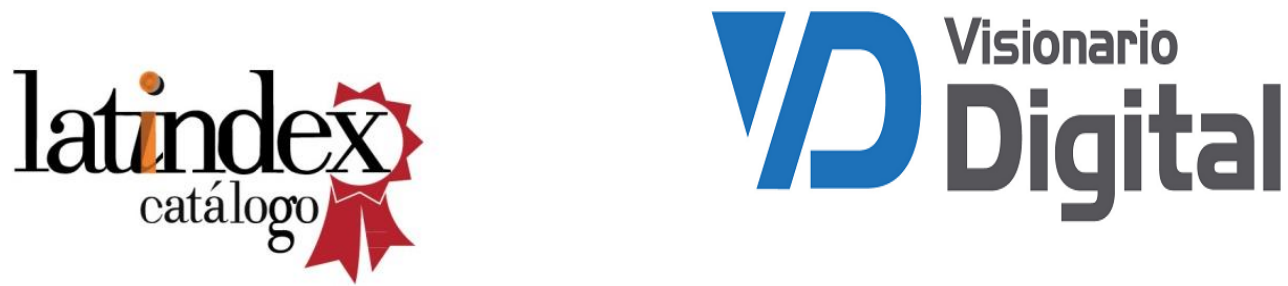